\title{
Delay, multiplicity, and non-existence of equilibrium in unanimity bargaining games
}

Citation for published version (APA):

Britz, V., Herings, P. J. J., \& Predtetchinski, A. (2015). Delay, multiplicity, and non-existence of equilibrium in unanimity bargaining games. Journal of Mathematical Economics, 61, 192-202. https://doi.org/10.1016/j.jmateco.2015.09.005

Document status and date:

Published: 01/12/2015

DOI:

10.1016/j.jmateco.2015.09.005

Document Version:

Publisher's PDF, also known as Version of record

Document license:

Taverne

\section{Please check the document version of this publication:}

- A submitted manuscript is the version of the article upon submission and before peer-review. There can be important differences between the submitted version and the official published version of record.

People interested in the research are advised to contact the author for the final version of the publication, or visit the DOI to the publisher's website.

- The final author version and the galley proof are versions of the publication after peer review.

- The final published version features the final layout of the paper including the volume, issue and page numbers.

Link to publication

\footnotetext{
General rights rights.

- You may freely distribute the URL identifying the publication in the public portal. please follow below link for the End User Agreement:

www.umlib.nl/taverne-license

Take down policy

If you believe that this document breaches copyright please contact us at:

repository@maastrichtuniversity.nl

providing details and we will investigate your claim.
}

Copyright and moral rights for the publications made accessible in the public portal are retained by the authors and/or other copyright owners and it is a condition of accessing publications that users recognise and abide by the legal requirements associated with these

- Users may download and print one copy of any publication from the public portal for the purpose of private study or research.

- You may not further distribute the material or use it for any profit-making activity or commercial gain

If the publication is distributed under the terms of Article $25 \mathrm{fa}$ of the Dutch Copyright Act, indicated by the "Taverne" license above, 


\title{
Delay, multiplicity, and non-existence of equilibrium in unanimity bargaining games
}

\author{
Volker Britz ${ }^{a}$, P. Jean-Jacques Herings ${ }^{\text {b,* }}$, Arkadi Predtetchinski ${ }^{b}$ \\ ${ }^{a}$ Center of Economic Research at ETH Zurich (CER-ETH), Switzerland \\ ${ }^{\mathrm{b}}$ Department of Economics, Maastricht University, Netherlands
}

\section{A R T I C L E I N F O}

\section{Article history:}

Received 16 April 2015

Received in revised form

24 July 2015

Accepted 16 September 2015

Available online 30 September 2015

\section{Keywords:}

Strategic bargaining

Subgame perfect equilibrium

Stationary strategies

Nash bargaining solution

\begin{abstract}
A B S T R A C T
We consider a class of perfect information bargaining games with unanimity acceptance rule. The proposer and the order of responding players are determined by the state that evolves stochastically over time. The probability distribution of the state in the next period is determined jointly by the current state and the identity of the player who rejected the current proposal. This protocol encompasses a vast number of special cases studied in the literature. We show that subgame perfect equilibria in pure stationary strategies need not exist. When such equilibria do exist, they may exhibit delay. Limit equilibria as the players become infinitely patient need not be unique.
\end{abstract}

(c) 2015 Elsevier B.V. All rights reserved.

\section{Introduction}

In his seminal paper, Rubinstein (1982) studies the division of a surplus among two impatient players through a non-cooperative bargaining game. Following this contribution, a rich literature has emerged which extends and generalizes Rubinstein's approach. A number of general results have persistently and recurrently emerged from this literature: in bargaining games where a surplus is divided under unanimity rule, equilibria exist, are efficient, and converge to a weighted Nash bargaining solution in the limit. In this paper, we explore the boundaries of the scope under which these general results are valid.

We consider bargaining games with the following characteristics. There is a finite number of players who need to make a unanimous choice for one particular payoff vector within a fulldimensional set of feasible payoffs. The game is set in discrete time and in each round of the game, one player is selected as the proposer. His role is to suggest one particular feasible payoff vector. The other players then sequentially accept or reject this proposal in some fixed order. If all players agree to the proposal, the game ends and the agreed upon payoffs are realized. As soon as one of the players rejects the current proposal, the game proceeds to the

\footnotetext{
* Corresponding author.

E-mail addresses: vbritz@ethz.ch (V. Britz), p.herings@maastrichtuniversity.nl (P.J.-J. Herings), a.predtetchinski@maastrichtuniversity.nl (A. Predtetchinski).

next round. We assume an exogenous breakdown of the negotiation to occur after each disagreement with probability $1-\delta$. Timediscounting and the possibility of an exogenous breakdown are largely interchangeable interpretations of $\delta$. The term "bargaining friction" can be used to capture both of them. The importance of the bargaining friction lies in the fact that it creates an incentive to come to an agreement sooner rather than later.

In order to complete the description of a unanimity bargaining game, one has to specify a rule which determines which player is the proposer in what round. We will refer to this rule as the protocol in the sequel. Rubinstein (1982) studies a game with only two players who simply take turns in making proposals, the alternating offer protocol. Rubinstein finds a unique subgame perfect equilibrium. The equilibrium strategies happen to be stationary.

It is well-known that the uniqueness of subgame perfect equilibrium breaks down in unanimity bargaining games with more than two players. With regard to those games, the literature focuses on subgame perfect equilibrium in pure stationary strategies (SSPE), which allow sharp predictions of the equilibrium payoffs at least when the discount factor is sufficiently close to one. Arguably the most obvious generalization of Rubinstein's alternating offer protocol to the case with more than two players is the rotating protocol, under which players become proposers in ascending order, and the first player proposes again after the last player. One alternative proper generalization of the alternating offers protocol is the rejector-proposes protocol introduced in Selten (1981) in a coalitional bargaining set-up. Under that rule, the 
first player to reject the current proposal becomes the next proposer. The rejector-proposes protocol is an example of an endogenous protocol in which the actions taken by the players throughout the game have an influence on the proposer selection. One important example of a protocol which is not a proper generalization of Rubinstein's alternating offers protocol is the time-invariant probability protocol which consists of an exogenously given probability distribution from which the proposer is drawn in each round.

The literature on unanimity bargaining games has established some results that are generally valid no matter which of these protocols is assumed.

1. An SSPE exists.

2. Every SSPE has no delay.

3. Every SSPE has efficient proposals.

4. There is a unique limit equilibrium as $\delta$ approaches one.

5. All limit equilibrium proposals are equal to a weighted Nash bargaining solution.

The aforementioned results follow from Britz et al. (2010) for exogenous protocols and from Britz et al. (2014) for endogenous protocols. They extend earlier work for more specific protocols by Binmore et al. (1986), Hart and Mas-Colell (1996), Kultti and Vartiainen (2010), Laruelle and Valenciano (2008), and Miyakawa (2008).

The weights in the Nash bargaining solution corresponding to the limit equilibrium proposals depend on the distribution of bargaining power inherent in the protocol. Kultti and Vartiainen (2010) show that the weights are all equal to each other under the rotating protocol. Miyakawa (2008) and Laruelle and Valenciano (2008) study the time-invariant probability protocol. In this case, the vector of bargaining weights is given by the time-invariant probability distribution. Britz et al. (2010) study a protocol where the selection of the proposer is described by a Markov process. That is, there are $n$ probability distributions on the $n$ players. The identity of the proposer in the current round determines which of the probability distributions is used to draw the proposer in the following round. This Markovian protocol is both a generalization of the time-invariant probability protocol and the rotating protocol. Then, the vector of bargaining weights is given by the stationary distribution of the Markov process.

Britz et al. (2014) study a class of endogenous protocols, thereby covering the rejector-proposes protocol. They consider protocols which consist of $n$ probability distributions on the $n$ players. The identity of the player who rejects the current proposal determines which of those probability distributions will be used to draw the following proposer. The vector of bargaining weights is shown to be proportional to the vector of probabilities with which the players propose after their own rejections.

In this paper, we present a class of unanimity bargaining games that allows for a rich family of bargaining protocols. This is achieved by introducing a finite set of states and for each state, conditional on the identity of the rejecting player, a vector of transition probabilities to the new states. The state determines the identity of the proposer and the order of the responses. It is easily verified that all the aforementioned protocols follow as special cases. The modeling approach is closely related to the one of Merlo and Wilson (1995). We are more general in allowing for endogenous protocols, i.e. the vector of transition probabilities may depend on the identity of the rejecting player. Motivated by our desire to study the effects of the bargaining protocol itself, we are less general in not allowing for the set of feasible payoffs to depend on the state.

We demonstrate that the results of the bargaining literature as enumerated above do not generalize, even when we require the set of feasible payoffs to correspond to the division of a unit surplus. We construct an example with three players and three states. A player is the proposer in his own state and the states corresponding to Players 2 and 3 are absorbing. Once Player 2 or Player 3 is selected as the proposer, he will remain the proposer forever. In state 1 , the protocol follows the rejector-proposes protocol after rejections by Players 2 or 3 . In the example, any SSPE predicts delay. SSPE proposals need not be efficient. In the example there is a continuum of SSPEs. Since the example is valid for arbitrarily high values of $\delta$, it is then used to show that limit equilibria are not unique, that there are two accumulation points of limit equilibrium utilities, and that limit equilibrium proposals may not be equal to each other. We show that the example is robust to perturbations of the transition probabilities.

The main intuition behind the example is that Players 2 and 3 capture the entire surplus in their own state. Since the protocol is of the rejector-proposes type in state 1, an SSPE with immediate acceptance requires Player 1 to offer both of them at least $\delta$. Since the total surplus is equal to one, this is clearly infeasible when $\delta$ is above one half. All SSPEs are therefore such that the offer by Player 1 in state 1 is rejected by one of the other players.

Yildiz (2003) studies the role of optimism in explaining bargaining delay. He has an example which is similar to ours in the sense that each of the responding players has a continuation payoff of $\delta$, so that immediate agreement is impossible when $\delta$ exceeds one half. In the example by Yildiz (2003), however, this result is driven by the fact that due to optimism each player believes that he will become the proposer in the next round with probability one. These beliefs are incompatible and therefore at least one player is wrong about the protocol. In contrast, the delay in our example is derived in a set-up where the bargaining protocol is common knowledge among the players.

Finally, we modify our leading example in a simple way. In state 1, we use the rejector-proposes protocol for Players 2 and 3 with probability one half and assume that Player 1 remains the proposer with the complementary probability. We demonstrate that now both SSPEs with immediate agreement and SSPEs with delay fail to exist. Herings and Predtetchinski (2015) have shown for the time-invariant probability protocol that an SSPE exists even when the set of feasible payoffs is non-convex. It follows that variations in the protocol are more problematic for fundamental properties like existence and efficiency of an SSPE than variations in the set of feasible payoffs.

Our results complement some of the examples of equilibrium delay and non-existence found in the literature. An example of an SSPE exhibiting delay has been given in Chatterjee et al. (1993) in the context of coalitional bargaining. Unlike the unanimity bargaining games considered here, in coalitional bargaining games a proposing player may choose to make an offer to a subset of the players. The approval of the proposal by all players in the chosen coalition is then sufficient for the proposal to pass. Also in a coalitional bargaining context, Bloch (1996) shows that SSPEs need not exist. Merlo and Wilson (1995) show that an SSPE may exhibit delay if the size of the cake changes stochastically over time. Jéhiel and Moldovanu (1995) show that delay can arise due to externalities. In addition to these examples, where delay arises in a complete and perfect information framework, there is a literature on bargaining delays when the parties are asymmetrically informed, see for instance the review by Ausubel et al. (2002).

The plan of the paper is as follows. We start by formally describing a class of unanimity bargaining games in Section 2. Section 3 summarizes the results in the literature regarding existence, immediate agreement, efficiency, and limit equilibria. Section 4 presents the example where an SSPE predicts delay, inefficiency, and non-uniqueness of the limit equilibrium. Section 5 shows the example to be robust to perturbations in the transition probabilities. We show in Section 6 that an SSPE may even fail to exist all together and Section 7 show this example to be robust to perturbations in the transition probabilities. Section 8 discusses the extension of the analysis to coalitional bargaining and Section 9 concludes. 


\section{Model}

We consider a non-cooperative bargaining game $G(N, V, S$, $\left.\iota, p^{0}, p, \delta\right)$, where $N=\{1, \ldots, n\}$ is the set of players and $V \subset \mathbb{R}^{n}$ is the set of feasible payoffs. Bargaining takes place in discrete time $t=0,1, \ldots$. In each round, one player is selected as the proposer and proposes an element $v$ of $V$. Next, the players sequentially respond to the proposal and, in case of unanimous acceptance, the proposal is implemented and the game ends with payoffs $v$ to the players. As soon as one player rejects, the game breaks down with probability $1-\delta$ and continues to the next round with probability $\delta \in(0,1)$. In case of breakdown, as well as in case of perpetual disagreement, payoffs to all players are equal to zero.

Our emphasis will be on the role of the protocol in determining the bargaining outcome. The set of feasible payoffs $V$ is therefore kept fixed in each round, but the bargaining protocol is allowed to be quite general. To achieve this, we make use of a finite state space $S$. The function $\iota: S \rightarrow N \times \Pi$, where $\Pi$ is the set of permutations on $N$, assigns to each state a proposer and an order of responders. That is, if $\iota(s)=(i, \pi)$, then Player $i$ is the proposer in state $s$ and all players sequentially respond to the proposal in the order $\pi(1), \ldots, \pi(n)$ given by the permutation $\pi$.

In round $t=0$, the initial state is determined by the probability distribution $p^{0} \in \Delta(S)$, where $\Delta(S)$ is the set of probability distributions on $S$. In any round $t>0$, the state of the game is determined by transition functions $p^{j}: S \rightarrow \Delta(S)$, one for each Player $j \in N$. If Player $j \in N$ rejects the proposal at time $t$ when the game is in state $s$, then $p^{j}(s)$ returns the probability distribution from which the state at time $t+1$ is drawn conditional on the continuation of the negotiations.

Many protocols that have been studied in the bargaining literature are special cases of the class of protocols described above, up to relatively unimportant modeling details. Such modeling details concern whether there is some probability of breakdown of negotiations, or whether players have time preferences. In case time preferences take the discounted utility form, although conceptually different from the risk preferences that are needed to study models with breakdown, both approaches lead to the same results as argued in Binmore et al. (1986). Another issue is whether players vote simultaneously or sequentially. Under simultaneous voting, the solution concept of subgame perfection has less bite and on top of subgame perfection it is typically required that players use stage-undominated voting strategies to avoid coordination problems. We study sequential voting in this paper. A final modeling detail is that we formally allow the proposer to turn down his own proposal.

The alternating offer protocol studied in the seminal contribution of Rubinstein (1982) corresponds to the case where $S=N=$ $\{1,2\}$, Player $s$ is the proposer in state $s$, and state transitions are such that the state alternates between periods. Player 1 is the initial proposer. Kultti and Vartiainen (2010) consider a multilateral extension of alternating offer bargaining, where proposers rotate in making offers. Their model corresponds to the case where $S=N$, Player $s$ is the proposer in state $s$, and the state transition is to state $s+1$ modulo $n$ with probability 1 if the current state is $s$. Binmore (1987) and Banks and Duggan (2000) consider the time-invariant probability protocol. This protocol results when $S=N$ and there is a fixed probability distribution $p^{0}$ on $S$ such that the proposer is selected in accordance with $p^{0}$ in every time period. Kalandrakis (2004) and Britz et al. (2010) consider the case where $S=N$ and require that for all $j, k \in N, p^{j}=p^{k}$. For $s \in S$, it holds that $\iota(s)=\left(s, \pi^{0}\right)$, where $\pi^{0}$ is the identity. The state denotes the current proposer and Player $i$ responds before Player $j$ if and only if $i<j$. State transitions are not influenced by the identity of the rejecting player, but are otherwise general, so this Markovian protocol includes the rotating protocol and the time-invariant probability protocol as special cases. Merlo and Wilson (1995) consider a general state space $S$ and assume that for all $j, k \in N, p^{j}=p^{k}$. They allow the set of feasible payoffs to depend on the state $s$, but since our attention here is on the influence of the protocol on the allocation of payoffs, we consider a fixed set $V$ instead.

All the bargaining protocols described in the previous paragraph have in common that the actions taken by the players are without consequence for the way the bargaining protocol proceeds in case of a rejection, i.e. for all $j, k \in N$ it holds that $p^{j}=p^{k}$. We refer to these protocols as exogenous.

The rejector-proposes protocol is introduced in Selten (1981) in a coalitional bargaining set-up and specifies that the player who rejects the current proposal is automatically called upon to make the next proposal. Kawamori (2008) generalizes this protocol to allow for a general probabilistic selection of a new proposer, conditional on who rejects the current proposal. When we apply his coalitional bargaining model to our unanimity bargaining setup, we obtain the case where $S=N$, for $s \in S$ it holds that $\iota(s)=\left(s, \pi^{0}\right)$, where $\pi^{0}$ is some fixed permutation of the players, and for all $s, s^{\prime} \in S, p^{j}(s)=p^{j}\left(s^{\prime}\right)$.

Whenever for some $j, k \in N, p^{j} \neq p^{k}$, the actions of the players influence the way the bargaining protocol proceeds, and we refer to such protocols as endogenous protocols. The rejector-proposes protocol is a key example of an endogenous protocol.

The state space $S$ can be used to model much more complicated protocols. For instance, a state could incorporate some finite history of past proposers or some finite history of past rejectors.

\section{Results in the existing literature}

Multilateral bargaining games are known to admit a wide multiplicity of subgame perfect equilibria, see Herrero (1985) and Haller (1986). It is therefore common in the literature to restrict attention to subgame perfect equilibria in pure stationary strategies. Although ideally the notion of stationarity should follow endogenously from the specification of the game as in Maskin and Tirole (2001), the literature typically takes the more ad hoc approach described below, which in general is weaker than the stationarity notion of Maskin and Tirole (2001).

A pure stationary strategy for Player $i$ in the game $G(\delta)$ consists, for each state $s \in S$ such that $\iota(s)=(i, \pi)$ for some $\pi \in \Pi$, of a proposal $\theta^{s} \in V$ and, for each state $s \in S$, of an acceptance set $A^{i, s} \subset V$. A stationary strategy of a player specifies a unique action for each of his decision nodes. This action depends only on the state and not on any other aspect of the history if the player is a proposer and on the state as well as the proposal made if the player is a responder. A stationary strategy profile $(\theta, A)$ leads to a unique probability distribution over payoffs in $V$, so determines the utility $u_{i}(\theta, A)$ of Player $i \in N$. Conditional utilities are denoted by $u_{i}(\theta, A \mid s)$. The social acceptance set in state $s \in S$ is defined as $A^{s}=\bigcap_{i \in N} A^{i, s}$. The social acceptance set consists of all alternatives that are unanimously accepted when proposed in state $s$.

Definition 3.1. A stationary subgame perfect equilibrium (SSPE) is a profile of pure stationary strategies that is a subgame perfect equilibrium of the game.

We would like to emphasize that since an SSPE is a subgame perfect equilibrium, no player has a profitable deviation from it, where a deviation is allowed to be non-stationary.

We make the following standard assumptions on $V$, where we use the notation $V_{+}=V \cap \mathbb{R}_{+}^{n}$ and $\partial V_{+}$for the set of weakly Pareto efficient points in $V_{+}$. Moreover, a vector $\eta$ with $\|\eta\|=1$ is said to be normal to the set $V$ at a point $\bar{v} \in V$ if $(v-\bar{v})^{\top} \eta \leq 0$ for every $v \in V$. The set of all vectors $\eta$ normal to $V$ at $\bar{v}$ is called the normal to $V$ at $\bar{v}$. 
Assumption A. The set $V$ is closed, convex, and comprehensive from below. The origin lies in the interior of $V$. The set $V_{+}$is bounded and all points in $\partial V_{+}$are strongly Pareto efficient. There is a unique vector in the normal to $V$ at every $v \in \partial V_{+}$.

A stationary strategy profile $(\theta, A)$ is said to have no delay if for every $s \in S$ it holds that $\theta^{s} \in A^{s}$. A stationary strategy profile $(\theta, A)$ is said to have efficient proposals if for every $s \in S$ it holds that $\theta^{s} \in \partial V_{+}$.

Apart from the analysis of $G(\delta)$, the literature also typically studies the behavior of equilibria when the continuation probability $\delta$ tends to 1 .

Definition 3.2. The profile of proposals $\bar{\theta}=\left(\bar{\theta}^{s}\right)_{s \in S}$ is a limit equilibrium if there is a sequence $\left\{\delta_{m}\right\}_{m \in \mathbb{N}}$ of continuation probabilities in $[0,1)$ converging to 1 and a sequence of profiles $\left\{\theta\left(\delta_{m}\right)\right\}_{m \in \mathbb{N}}=$ $\left\{\left(\theta^{s}\left(\delta_{m}\right)_{s \in S}\right)\right\}_{m \in \mathbb{N}}$, where $\theta\left(\delta_{m}\right)$ is an SSPE profile of proposals of the game $G\left(\delta_{m}\right)$, such that $\lim _{m \rightarrow \infty} \theta\left(\delta_{m}\right)=\bar{\theta}$.

A limit equilibrium is a profile of proposals that can be approximated arbitrarily close by an SSPE profile of proposals when the probability of breakdown is arbitrarily small. Of particular interest is the relationship between limit equilibria and the asymmetric Nash bargaining solution with positive weights $\mu \in \mathbb{R}_{+}^{n} \backslash\{0\}$, denoted $\mu$-ANBS and defined as follows.

Definition 3.3. The asymmetric Nash product with weights $\mu \in$ $\mathbb{R}_{+}^{n} \backslash\{0\}$ is the function $f: V_{+} \rightarrow \mathbb{R}$ defined by

$f(v)=\prod_{i \in N}\left(v_{i}\right)^{\mu_{i}}$.

The $\mu$-ANBS is the unique maximizer of the function $f$ on the set $V_{+}$.

Britz et al. (2010) study the class of exogenous protocols characterized by the following assumption.

Assumption B. It holds that $S=N$, for every $s \in S, \iota(s)=\left(s, \pi^{0}\right)$ with $\pi^{0}$ the identity, for all $j, k \in N, p^{j}=p^{k}$, and the matrix $M=\left[p^{j}(1) \cdots p^{j}(n)\right]$ is irreducible.

An irreducible matrix $M$ has a unique stationary distribution $\mu$. Recall that a stationary distribution $\mu$ is a probability distribution on the set of states satisfying $M \mu=\mu$. The Markovian protocols satisfying Assumption B are sufficiently rich to encompass the alternating offer protocol, the rotating protocol, and the timeinvariant probability protocol.

Britz et al. (2014) study the class of endogenous protocols characterized by the following assumption.

Assumption C. It holds that $S=N$, for every $s \in S, \iota(s)=\left(s, \pi^{0}\right)$ with $\pi^{0}$ the identity, for every $j \in N$, for every $s, s^{\prime} \in S, p^{j}(s)=$ $p^{j}\left(s^{\prime}\right)$. There exists $i \in N$ such that $p_{i}^{i}(i)>0$.

In Britz et al. (2014), the authors assume for the sake of simplicity that players respond to a proposal in the fixed order $1, \ldots, n$. As remarked in footnote 6 of that paper, all results would carry over to the case with arbitrary voting orders. We would like to emphasize that the matrix $M^{\prime}=\left[p^{1}(s) \cdots p^{n}(s)\right]$ is not assumed to be irreducible. The only requirement made in Assumption $C$ is that it has at least one non-zero diagonal element.

We associate to each protocol satisfying Assumption $C$ the weights $\mu>0$ given by $\mu_{j}=p_{j}^{j}(j), j \in N$, so $\mu_{j}$ is the probability that Player $j$ becomes the next proposer conditional on a rejection. The class of protocols satisfying Assumption C corresponds to the class studied in Kawamori (2008) and includes the rejectorproposes protocol as a special case.

The following result follows from Britz et al. (2010, 2014).
Theorem 3.4. If Assumptions $\mathrm{A}$ and $\mathrm{B}$, or Assumptions $\mathrm{A}$ and $\mathrm{C}$ are satisfied, then

1. An SSPE exists.

2. Every SSPE has no delay.

3. Every SSPE has efficient proposals.

4. There is a unique limit equilibrium.

5. All limit equilibrium proposals are equal to the $\mu$-ANBS.

The characterization of limit equilibrium proposals as a weighted Nash bargaining solution has been shown in Binmore et al. (1986) for bilateral bargaining. For multilateral bargaining, this result is obtained in Hart and Mas-Colell (1996) for the time-invariant probability protocol with uniform recognition probabilities and in Miyakawa (2008) and Laruelle and Valenciano (2008) for general recognition probabilities. Kultti and Vartiainen (2010) derive this result for the rotating offer protocol. Theorem 3.4 includes these results as special cases. The five claims of Theorem 3.4 and in particular the limit equilibrium payoffs are independent of the order in which the responding players accept or reject the proposal. This is noteworthy in the case of an endogenous protocol. For instance, one might have conjectured that the rejector-proposes protocol favors the player who comes first in the responder order.

Ideally one would like to prove Theorem 3.4 for the entire class of bargaining protocols as laid down in Section 2, up to standard regularity assumptions on the set of feasible payoffs as in Assumption A and under appropriate regularity assumptions on the protocol that generalize Assumptions B and C to the setup of this paper. Assumption D presents a strong version of such regularity assumptions.

Assumption D. For every $i \in N$, there exists $s \in S$ and $\pi \in \Pi$ such that $\iota(s)=(i, \pi)$. For every $s, s^{\prime} \in S$, for every $i \in N, p_{s^{\prime}}^{i}(s)>0$.

The first part of the assumption says that every player is the proposer in some state. The second part says that for every player, for every pair of states, there is positive probability that the player becomes the proposer in one state if he rejects a proposal in the other state. For the model with $S=N$ and rejector-independent transition probabilities, Assumption D implies Assumption B. For the model with $S=N$ and state-independent transition probabilities, Assumption D implies Assumption C. In view of Theorem 3.4, one could then conjecture that the five results stated in Theorem 3.4 hold in our model if Assumptions A and D are satisfied. However, we will show in the sequel that this is not true. In particular, in Proposition 5.3, we will show that a bargaining protocol can satisfy Assumption D but still violate the no delay and efficient proposals properties of Theorem 3.4.

\section{Failure of Properties 2, 3, 4, and 5}

In this section, we present an example where Properties 2, 3, 4 , and 5 of Theorem 3.4 are violated. Moreover, the example is minimal in the following sense: It has $S=N=\{1,2,3\}$, and for two states out of three we have $p^{j}(s)=p^{k}(s)$, for all $j, k \in N$. With the exception of one state, the protocol therefore satisfies Assumption B and is exogenous.

Example 4.1. There are three players and three states, $S=N=$ $\{1,2,3\}$. Each player is the proposer in one state and players respond in ascending order, so we have

$\iota(1)=\left(1, \pi^{0}\right)$,

$\iota(2)=\left(2, \pi^{0}\right)$,

$\iota(3)=\left(3, \pi^{0}\right)$, 
where $\pi^{0}$ is the identity. Players have to divide a surplus of one unit, $V=\left\{v \in \mathbb{R}^{3} \mid v_{1}+v_{2}+v_{3} \leq 1\right\}$. This set clearly satisfies Assumption A. In state $s=1$, we follow the rejector-proposes protocol for Players 2 and 3. If Player 1 rejects his own proposal in state $s=1$, then each of the three states is selected next with equal probability.

$p^{1}(1)=\left(\frac{1}{3}, \frac{1}{3}, \frac{1}{3}\right)$

$p^{2}(1)=(0,1,0)$,

$p^{3}(1)=(0,0,1)$.

States $s=2,3$ are absorbing.

$p^{i}(2)=(0,1,0), \quad i \in N$,

$p^{i}(3)=(0,0,1), \quad i \in N$.

The next result claims not only that equilibria may exhibit delay, but even makes the stronger statement that all SSPEs feature delay for sufficiently high values of $\delta$.

Proposition 4.2. For $\delta>1 / 2$, every SSPE in Example 4.1 has delay.

Proof. Suppose by way of contradiction that $(\theta, A)$ is an SSPE which has no delay. Consider a subgame starting with a proposal by Player 2 in state 2 . In this subgame, Player 2 remains the proposer forever, and it is straightforward to verify that the subgame has a unique SSPE where Player 2 captures the entire surplus. It holds that $u(\theta, A \mid 2)=(0,1,0)$. By a completely symmetric argument, we find that $u(\theta, A \mid 3)=(0,0,1)$. Now consider a subgame starting with a proposal by Player 1 in state 1 . Since $(\theta, A)$ has no delay, Player 2 accepts $\theta^{1}$ and it holds that $\theta_{2}^{1} \geq \delta$, since a rejection by Player 2 leads to a breakdown with probability $1-\delta$ and a transition to state 2 and a payoff of 1 for Player 2 with probability $\delta$. Similarly, it holds that $\theta_{3}^{1} \geq \delta$, since a rejection by Player 3 leads to a breakdown with probability $1-\delta$ and a transition to state 3 and a payoff of 1 for Player 3 with probability $\delta$. It follows that

$\theta_{1}^{1} \leq 1-\theta_{2}^{1}-\theta_{3}^{1} \leq 1-2 \delta<0$.

Since Player 1 can ensure a non-negative payoff by a strategy that rejects all proposals, we have obtained a contradiction to $(\theta, A)$ being an SSPE which has no delay.

The intuition behind the example is the following. If Player 2 rejects the proposal of Player 1 , then the game goes to an absorbing state where Player 2 remains the proposer forever. It is well-known that in any SSPE of such a subgame, Player 2 would capture the entire surplus. Thus, when the game is in state 1, Player 2 can guarantee himself a payoff of $\delta$ by rejecting Player 1's proposal. In any SSPE with no delay, Player 1 would need to offer at least the amount $\delta$ to Player 2. The same argument applies to Player 3: If Player 3 rejects a proposal of Player 1, the game goes to an absorbing state where Player 3 remains the proposer forever and can capture the entire surplus. Thus, when Player 3 reacts to the proposal of Player 1, he will not accept any less than $\delta$. Indeed, the sum of the responding players' reservation payoffs is equal to $2 \delta$. We can see that if $\delta>\frac{1}{2}$, then the available surplus is not sufficient for Player 1 to pay the other two players their reservation payoffs. Consequently, if $\delta>\frac{1}{2}$, no agreement can be reached in state 1 .

The next issue is whether there is an SSPE with delay in Example 4.1 for $\delta>1 / 2$. Consider a strategy profile $(\bar{\theta}, \bar{A})$ with proposals $\bar{\theta}^{1} \in V, \bar{\theta}^{2}=(0,1,0)$, and $\bar{\theta}^{3}=(0,0,1)$, and acceptance sets

$\bar{A}^{1, s}=\left\{v \in V \mid v_{1} \geq 0\right\}, \quad s=1,2,3$,

$\bar{A}^{2,1}=\left\{v \in V \mid v_{2} \geq \delta, v_{3} \geq \delta\right\}$,

$\bar{A}^{2,2}=\left\{v \in V \mid v_{2} \geq \delta\right\}$,
$\bar{A}^{2,3}=\left\{v \in V \mid v_{2} \geq 0\right\}$,

$\bar{A}^{3, s}=\left\{v \in V \mid v_{3} \geq \delta\right\}, \quad s=1,3$,

$\bar{A}^{3,2}=\left\{v \in V \mid v_{3} \geq 0\right\}$.

When players play according to $(\bar{\theta}, \bar{A})$, Player 1 makes a particular proposal belonging to $V$ in state 1 , which will be rejected by some player when $\delta>1 / 2$. More precisely, the proposal $\bar{\theta}^{1}$ in state 1 is rejected by Player 1 when $\bar{\theta}_{1}^{1}<0$ and is rejected by Player 2 otherwise. Notice that in state 1, Player 2 would even reject the proposal $(0,1,0)$, since acceptance of such a proposal would lead to a rejection by Player 3 , followed by breakdown of the negotiations or a transition to state 3 .

Consider first the case where $\bar{\theta}_{1}^{1}<0$. Player 1 now rejects his own proposal, negotiations break down with probability $1-\delta$ and continue with probability $\delta$. If negotiations continue, transitions occur with equal probability to each of the three states, a rejection of proposal $\bar{\theta}^{1}$ by Player 1 in state 1 , an acceptance of payoff vector $(0,1,0)$ in state 2 , and an acceptance of payoff vector $(0,0,1)$ in state 3.

Consider next the situation where $\bar{\theta}_{1}^{1} \geq 0$. Since $\bar{\theta}_{1}^{1} \geq 0$ and $\delta>1 / 2$, Player 2 rejects the proposal, negotiations break down with probability $1-\delta$ and continue in state 2 with probability $\delta$. In the latter case, the payoff vector $(0,1,0)$ is proposed and accepted.

Proposition 4.3. For $\delta>1 / 2$, the strategy profile $(\bar{\theta}, \bar{A})$ is an SSPE in Example 4.1.

Proof. To show that $(\bar{\theta}, \bar{A})$ is an SSPE, it suffices to verify the oneshot deviation property, see for instance Fudenberg and Tirole (1991). We consider three cases, depending on the state to which a decision node belongs.

Case 1. Decision nodes in state 1 .

After a history in state 1 where Player 1 has to propose, the proposal $\bar{\theta}^{1}$ is rejected, either by Player 1 in case $\bar{\theta}_{1}^{1}<0$ or by Player 2 in case $\bar{\theta}_{1}^{1} \geq 0$, and leads ultimately to breakdown, or the acceptance of proposal $\bar{\theta}^{2}$, or the acceptance of proposal $\bar{\theta}^{3}$. In all cases, Player 1 receives a payoff of zero. A one-shot deviation to any other proposal is rejected as well, either by Player 1 or by Player 2, and also leads ultimately to a payoff of zero for sure. Such a deviation is therefore not profitable.

After a history in state 1 where Player 1 has to respond, any proposal $v$ with $v_{1}<0$ is rejected by Player 1 , and ultimately leads to a payoff of zero for sure. A one-shot deviation to acceptance leads to the acceptance of $v$ and a negative payoff for Player 1 , or the rejection of $v$ by Player 2 or Player 3 and a payoff of zero for Player 1 . Such a deviation is therefore not profitable. Any proposal $v$ with $v_{1} \geq 0$ is accepted by Player 1 , next rejected by Player 2 , and followed by breakdown of the negotiations or acceptance of $(0,1,0)$ in the next period. The payoff for Player 1 is therefore zero. A one-shot deviation to rejection leads ultimately to a payoff of zero for Player 1 as well and is therefore not profitable.

After a history in state 1 where Player 2 has to respond, any proposal $v$ with $v_{2}<\delta$ or $v_{3}<\delta$ is rejected by Player 2, which results in a payoff of $\delta$ for Player 2. A one-shot deviation to acceptance is followed by an acceptance by Player 3 if $v_{3} \geq \delta$ and leads to payoff $v_{2}<\delta$ for Player 2, so is not profitable, and is followed by a rejection by Player 3 if $v_{3}<\delta$, leading to payoff 0 for Player 2, so is not profitable either. Any proposal $v$ with $v_{2} \geq \delta$ and $v_{3} \geq \delta$ is accepted by Player 2 , followed by an acceptance by Player 3 , and a payoff of $v_{2}$ for Player 2 . A one-shot deviation to rejection leads to a payoff of $\delta \leq v_{2}$ for Player 2 and is therefore not profitable.

After a history in state 1 where Player 3 has to respond, any proposal $v$ with $v_{3}<\delta$ is rejected by Player 3 , resulting in a payoff of $\delta$ for Player 3. A one-shot deviation to acceptance is clearly not profitable. Any proposal $v$ with $v_{3} \geq \delta$ is accepted by Player 3 , 
leading to a payoff of $v_{3}$ for Player 3. A one-shot deviation to rejection is clearly not profitable.

Case 2. Decision nodes in state 2.

After a history in state 2 where Player 2 has to propose, the proposal $\bar{\theta}^{2}=(0,1,0)$ by Player 2 is accepted by all players, and leads to utility 1 for Player 2 . Since Players 1 and 3 reject proposals which give them a negative payoff, there are no profitable one-shot deviations for Player 2. Since a one-shot rejection by any player leads to payoffs $(0, \delta, 0)$, the one-shot deviation property holds for responders.

Case 3. Decision nodes in state 3.

This case is similar to Case 2.

Proposition 4.3 describes a continuum of SSPEs, parametrized by the proposal $\bar{\theta}^{1}$ by Player 1 . For equilibria with $\bar{\theta}_{1}^{1}<0$, the equilibrium payoffs when starting in state 1 are equal to $u(\bar{\theta}, \bar{A}$ | $1)=(0, \delta /(3-\delta), \delta /(3-\delta))$. For equilibria with $\theta_{1}^{1} \geq 0$, it holds that $u(\bar{\theta}, \bar{A} \mid 1)=(0, \delta, 0)$. None of the properties, apart from SSPE existence, mentioned in Theorem 3.4 are satisfied. All SSPEs have delay. There is a continuum of SSPEs where Player 1 makes an inefficient proposal, and even if Player 1 makes an efficient proposal, it is still rejected by Player 1 or Player 2 . Any element of $V \times\{(0,1,0)\} \times\{(0,0,1)\}$ can be a limit equilibrium proposal, so there is no unique limit equilibrium. When starting in state 1 , limit equilibrium utilities are either equal to $(0,1 / 2,1 / 2)$ or $(0,0,1)$. Finally, limit equilibrium proposals are not equal to each other.

\section{Robustness of Example 4.1}

In this section, we will examine the robustness of Example 4.1 to perturbations of the transition probabilities. In particular, we will see that the presence of absorbing states is not vital for equilibrium delay, inefficient proposals, multiple limit equilibria, and limit equilibrium proposals that are not equal to each other.

Indeed, one may object to Example 4.1 that states 2 and 3 are absorbing, no matter what actions the players take, and therefore Assumption D is violated. Still, we would like to argue that Example 4.1 is robust to perturbations in the transition probabilities and that the violations of Properties 2, 3, 4, and 5 of Theorem 3.4 are not due to the presence of absorbing states. Indeed, we will now present a perturbed version of Example 4.1 which satisfies Assumption D and show that nevertheless the Properties 2, 3, 4, and 5 of Theorem 3.4 are violated. To be more specific, consider the case where all the transition probabilities are perturbed by some $\varepsilon \in(0,1 / 3)$. We obtain the following example.

Example 5.1. There are three players and three states, $S=N=$ $\{1,2,3\}$. Each player is the proposer in one state and players respond in ascending order, so we have

$\iota(1)=\left(1, \pi^{0}\right)$,

$\iota(2)=\left(2, \pi^{0}\right)$,

$\iota(3)=\left(3, \pi^{0}\right)$,

where $\pi^{0}$ is the identity. Players have to divide a surplus of one unit, $V=\left\{v \in \mathbb{R}^{3} \mid v_{1}+v_{2}+v_{3} \leq 1\right\}$. In state $s=1$, the transitions depend on the identity of the player who rejects a proposal. If Player 1 rejects his own proposal in state $s=1$, then each of the three states is selected next with equal probability. If Player $j=2,3$ rejects the proposal by Player 1 , then we move to the state in which $j$ is proposer with probability $1-2 \varepsilon$. If we do not go to the state in which player $j$ proposes, then one of the other two states is chosen randomly.

$p^{1}(1)=\left(\frac{1}{3}, \frac{1}{3}, \frac{1}{3}\right)$, $p^{2}(1)=(\varepsilon, 1-2 \varepsilon, \varepsilon)$,

$p^{3}(1)=(\varepsilon, \varepsilon, 1-2 \varepsilon)$.

In states $s=2,3$, the transitions are independent of the identity of the rejecting player. The process returns to the same state with probability $1-2 \varepsilon$. The process continues with probability $\varepsilon$ in each of the other two states.

$\begin{array}{ll}p^{i}(2)=(\varepsilon, 1-2 \varepsilon, \varepsilon), & i \in N, \\ p^{i}(3)=(\varepsilon, \varepsilon, 1-2 \varepsilon), & i \in N .\end{array}$

Notice that the transition dynamics in this example satisfy Assumption D. In particular, each player is the proposer in one of the three states. Moreover, the example is such that regardless of the current state and the identity of the rejector, we move with positive probability to every state after every rejection.

Consider a strategy profile $(\hat{\theta}, \hat{A})$ with proposals $\hat{\theta}^{1} \in\{v \in V$ | $\left.v_{1}<0\right\}$,

$\hat{\theta}^{2}=(0,1-y, y)$,

$\hat{\theta}^{3}=(0, y, 1-y)$,

where

$y=\frac{3 \delta \varepsilon}{(3-\delta)(1-\delta+3 \delta \varepsilon)}$,

and acceptance sets

$\hat{A}^{1, s}=\left\{v \in V \mid v_{1} \geq 0\right\}, \quad s=1,2,3$,

$\hat{A}^{2,1}=\left\{v \in V \mid v_{2} \geq z, v_{3} \geq z\right\}$,

$\hat{A}^{2,2}=\left\{v \in V \mid v_{2} \geq z\right\}$,

$\hat{A}^{2,3}=\left\{v \in V \mid v_{2} \geq y\right\}$,

$\hat{A}^{3, s}=\left\{v \in V \mid v_{3} \geq z\right\}, \quad s=1,3$,

$\hat{A}^{3,2}=\left\{v \in V \mid v_{3} \geq y\right\}$,

where

$z=\frac{\delta(1-\delta)\left(3-\delta-9 \delta \varepsilon^{2}\right)+\delta \varepsilon(2-\delta)(6 \delta-3)}{(3-\delta)(1-\delta+3 \delta \varepsilon)}$.

Notice that if $\varepsilon$ tends to zero, then $y$ tends to zero and $z$ to $\delta$.

When players play according to $(\hat{\theta}, \hat{A})$, Player 1 makes a proposal $\hat{\theta}^{1}$ in $V$ with $\hat{\theta}_{1}^{1}<0$ in state 1 , which is rejected by Player 1 himself, and a transition to each of the three states follows with equal probability. In state 2, Player 2 makes a proposal that gives a payoff of 0 to Player 1, gives the reservation payoff $y$ to Player 3, and keeps the remainder of the surplus himself. State 3 is symmetric to state 2 , with the roles of Players 2 and 3 reversed. The proposals $\hat{\theta}^{2}$ and $\hat{\theta}^{3}$ in states 2 and 3 are accepted since $1-y>z$, which follows from the fact that

$$
\begin{aligned}
(3-\delta)(1-\delta)+3 \delta \varepsilon(2-\delta)> & \delta(1-\delta)\left(3-\delta-9 \delta \varepsilon^{2}\right) \\
& +\delta \varepsilon(2-\delta)(6 \delta-3) .
\end{aligned}
$$

Proposition 5.2. For every $\varepsilon \in(0,1 / 6)$, there exists $\bar{\delta}<1$ such that for every $\delta \geq \bar{\delta}$ the strategy profile $(\hat{\theta}, \hat{A})$ is an SSPE in Example 5.1.

Proof. For $s=1,2,3$, we define the equilibrium utilities conditional on state $s, x^{s}=u(\hat{\theta}, \hat{A} \mid s)$. The symmetry of the game and the strategies implies that $x_{2}^{1}=x_{3}^{1}, x_{1}^{2}=x_{1}^{3}, x_{2}^{2}=x_{3}^{3}$, and $x_{3}^{2}=x_{2}^{3}$. It holds that $x^{1}=(0, \delta /(3-\delta), \delta /(3-\delta)), x^{2}=\hat{\theta}^{2}$, and $x^{3}=\hat{\theta}^{3}$, where the expression for $x^{1}$ uses the observation that $x_{2}^{1}=(\delta / 3)+(\delta / 3) x_{2}^{1}$. 
To show that $(\hat{\theta}, \hat{A})$ is an SSPE, we verify the one-shot deviation property. We consider three cases, depending on the state to which a decision node belongs.

Case 1. Decision nodes in state 1 .

Consider a history in state 1 after which Player 3 has to respond. A rejection followed by play according to $(\hat{\theta}, \hat{A})$ leads to a payoff for Player 3 equal to $\delta \varepsilon x_{3}^{1}+\delta \varepsilon x_{3}^{2}+\delta(1-2 \varepsilon) x_{3}^{3}$. A straightforward, but tedious, calculation reveals that this payoff is equal to $z$. Since Player 3 accepts proposals in state 1 if and only if $v_{3} \geq z$, this shows that the one-shot deviation property is satisfied.

Consider a history in state 1 after which Player 2 has to respond to a proposal $v$. Suppose first that $v_{3} \geq z$. A calculation similar to that in the previous paragraph shows that rejection of $v$ by Player 2 yields a payoff of $z$. Acceptance of $v$ by Player 2 leads to the payoff $v_{2}$ since $v$ is also accepted by Player 3 . We conclude that accepting the proposal $v$ if and only if $v_{2} \geq z$ does not violate the one-shot deviation principle.

Suppose now that $v_{3}<z$. As before, rejecting $v$ yields Player 2 a payoff of $z$. If Player 2 accepts $v$, it is rejected by Player 3 , yielding a continuation utility equal to $\delta \varepsilon x_{2}^{1}+\delta \varepsilon x_{2}^{2}+\delta(1-2 \varepsilon) x_{2}^{3}$. A straightforward, though tedious, calculation reveals the latter expression to be equal to $y$, and it holds that $y<z$ since

$z-y=\frac{\delta(1-\delta)(1-3 \varepsilon)(3-\delta+3 \delta \varepsilon)}{(3-\delta)(1-\delta+3 \delta \varepsilon)}>0$.

Hence $v$ should be rejected by Player 2 .

The verification of the one-shot deviation property for Player 1 is trivial for histories where he responds. Consider a history where Player 1 proposes. Player 1 has a profitable one-shot deviation if and only if he can make a proposal that gives more than $z$ to Players 2 and 3 and a positive payoff to himself. This implies that Player 1 has no profitable one-shot deviation if $2 z \geq 1$. This inequality is satisfied if and only if $\delta \geq 1 /(2-6 \varepsilon)$. It follows that for every $\varepsilon \in(0,1 / 6)$, there exists $\overline{\bar{\delta}}<1$ such that for every $\delta \geq \bar{\delta}, 2 z \geq 1$. Case 2. Decision nodes in state 2 .

Player 3 accepts a proposal $v$ if and only if $v_{3} \geq y$, where $y$ equals the continuation payoff of Player 3 following his rejection. This shows that the one-shot deviation property is satisfied.

Player 2 accepts a proposal $v$ if and only if $v_{2} \geq z$, where $z$ equals the continuation payoff of Player 2 following his rejection. We observe that acceptance of $v$ yields Player 2 payoff $v_{2}$ if Player 3 accepts as well, and $z$ if Player 3 rejects $v$. This shows that the oneshot deviation property is satisfied.

The verification of the one-shot deviation property for Player 1 is trivial.

Consider a history in state 2 after which Player 2 proposes. Since the proposal $\hat{\theta}^{2}$ of Player 2 gives Players 1 and 3 the least amount they are willing to accept, there is no profitable one-shot deviation for Player 2 which will be accepted by Players 1 and 3. Consider a one-shot deviation by Player 2 which is rejected by some player. Ultimately, such a proposal leads to breakdown and payoff 0 for Player 2, or an acceptance of $\hat{\theta}^{2}$ and payoff $1-y$ for Player 2 or an acceptance of $\hat{\theta}^{3}$ and Payoff $y$ for Player 2. Since $y<1-y$, the expected payoff for Player 2 is less than $x_{2}^{2}=1-y$, so the deviation is not profitable.

Case 3. Decision nodes in state 3.

By symmetry, the line of argument is the same as in Case 2 .

The equilibrium presented in Proposition 5.2 clearly violates Properties 2 and 3 of Theorem 3.4: No agreement is reached in state 1 , and the proposal made in state 1 need not be efficient. The equilibrium also has the remarkable feature that Player 1's equilibrium payoff is equal to zero in every state. This is surprising, since in the framework of Britz et al. (2014), the bargaining power of a player is proportional to the probability to propose conditional on his own rejection. In the protocol of Example 5.1, this probability is at least $\varepsilon$ for Player 1. Proposition 5.2 also gives us a multitude of limit equilibria, so that Properties 4 and 5 in Theorem 3.4 are also violated. More specifically, we can see that any point $\left(\theta^{1}, \theta^{2}, \theta^{3}\right)$ where $\theta^{1} \in V$ with $\theta_{1}^{1} \leq 0$ and $\theta^{2}=\theta^{3}=$ $\left(0, \frac{1}{2}, \frac{1}{2}\right)$ is a limit equilibrium.

Consider a strategy profile $(\tilde{\theta}, \tilde{A})$ with proposals $\tilde{\theta}^{1} \in\{v \in V \mid$ $\left.v_{1} \geq 0\right\}$,

$\tilde{\theta}^{2}=\left(0,1-y_{3}, y_{3}\right)$,

$\tilde{\theta}^{3}=\left(0, y_{2}, 1-y_{2}\right)$,

where

$y_{2}=\frac{\delta \varepsilon+\delta^{2} \varepsilon-3 \delta^{2} \varepsilon^{2}}{1-\delta+2 \delta \varepsilon+\delta^{2} \varepsilon-3 \delta^{2} \varepsilon^{2}}$,

$y_{3}=\frac{\delta \varepsilon}{1-\delta+2 \delta \varepsilon+\delta^{2} \varepsilon-3 \delta^{2} \varepsilon^{2}}$,

and acceptance sets

$\tilde{A}^{1, s}=\left\{v \in V \mid v_{1} \geq 0\right\}, \quad s=1,2,3$,

$\tilde{A}^{2,1}=\left\{v \in V \mid v_{2} \geq z_{2}, v_{3} \geq z_{3}\right\}$,

$\tilde{A}^{2,2}=\left\{v \in V \mid v_{2} \geq z_{2}\right\}$,

$\tilde{A}^{2,3}=\left\{v \in V \mid v_{2} \geq y_{2}\right\}$,

$\tilde{A}^{3, s}=\left\{v \in V \mid v_{3} \geq z_{3}\right\}, \quad s=1,3$,

$\tilde{A}^{3,2}=\left\{v \in V \mid v_{3} \geq y_{3}\right\}$,

where

$z_{2}=\frac{\delta-\delta^{2}-2 \delta \varepsilon+4 \delta^{2} \varepsilon-3 \delta^{2} \varepsilon^{2}}{1-\delta+2 \delta \varepsilon+\delta^{2} \varepsilon-3 \delta^{2} \varepsilon^{2}}$,

$z_{3}=\frac{\delta-\delta^{2}-2 \delta \varepsilon+3 \delta^{2} \varepsilon}{1-\delta+2 \delta \varepsilon+\delta^{2} \varepsilon-3 \delta^{2} \varepsilon^{2}}$.

Notice that if $\varepsilon$ tends to zero, then $y_{2}$ and $y_{3}$ tend to zero and $z_{2}$ and $z_{3}$ tend to $\delta$.

When players play according to $(\tilde{\theta}, \tilde{A})$, Player 1 makes a proposal $\tilde{\theta}^{1}$ in $V$ with $\tilde{\theta}_{1}^{1} \geq 0$ in state 1 . A straightforward calculation shows that $z_{2}+z_{3}>1$ if and only if

$(1-\delta)(2 \delta-6 \delta \varepsilon-1)>0$.

Therefore it holds that if $\varepsilon<1 / 6$ and $\delta>1 /(2-6 \varepsilon)$, then Player 2 rejects $\tilde{\theta}^{1}$, and a transition to state 2 follows with high probability. In state 2, Player 2 makes a proposal that gives a payoff of 0 to Player 1, gives the reservation payoff $y_{2}$ to Player 3 , and keeps the remainder of the surplus himself. State 3 is similar, with the roles of Players 2 and 3 reversed. The proposals $\tilde{\theta}^{2}$ and $\tilde{\theta}^{3}$ in states 2 and 3 are accepted since $1-y_{3} \geq z_{2}$ and $1-y_{2} \geq z_{3}$ which follows respectively from

$$
\begin{aligned}
1-\delta+\delta \varepsilon+\delta^{2} \varepsilon-3 \delta^{2} \varepsilon^{2} & >\delta(1-\delta)+2 \delta^{2} \varepsilon-3 \delta^{2} \varepsilon^{2} \\
& >\delta-\delta^{2}-2 \delta \varepsilon+4 \delta^{2} \varepsilon-3 \delta^{2} \varepsilon^{2}, \\
1-\delta+\delta \varepsilon>\delta-\delta^{2}-2 \delta \varepsilon & +3 \delta^{2} \varepsilon .
\end{aligned}
$$

Proposition 5.3. For every $\varepsilon \in(0,1 / 6)$, there exists $\bar{\delta}<1$ such that for every $\delta \geq \bar{\delta}$ the strategy profile $(\tilde{\theta}, \tilde{A})$ is an SSPE in Example 5.1.

Proof. For $s=1,2,3$, we define the equilibrium utilities conditional on state $s, x^{s}=u(\tilde{\theta}, \tilde{A} \mid s)$. We have that $x^{2}=\tilde{\theta}^{2}, x^{3}=\tilde{\theta}^{3}$, $x_{1}^{1}=0, x_{2}^{1}=\delta \varepsilon x_{2}^{1}+\delta(1-2 \varepsilon) x_{2}^{2}+\delta \varepsilon x_{2}^{3}$, so

$x_{2}^{1}=\frac{\delta-\delta^{2}-2 \delta \varepsilon+4 \delta^{2} \varepsilon-3 \delta^{2} \varepsilon^{2}}{1-\delta+2 \delta \varepsilon+\delta^{2} \varepsilon-3 \delta^{2} \varepsilon^{2}}$, 
and $x_{3}^{1}=\delta \varepsilon x_{3}^{1}+\delta(1-2 \varepsilon) x_{3}^{2}+\delta \varepsilon x_{3}^{3}$, so

$x_{3}^{1}=\frac{\delta \varepsilon}{1-\delta+2 \delta \varepsilon+\delta^{2} \varepsilon-3 \delta^{2} \varepsilon^{2}}$.

To show that $(\tilde{\theta}, \tilde{A})$ is an SSPE, we verify the one-shot deviation property. We consider three cases, depending on the state to which a decision node belongs.

Case 1. Decision nodes in state 1.

The following argument is analogous to the argument in the proof of the previous proposition, except that $z_{2}, z_{3}$ now differ.

Consider a history in state 1 after which Player 3 has to respond. A rejection followed by play according to $(\tilde{\theta}, \tilde{A})$ leads to a payoff for Player 3 equal to $\delta \varepsilon x_{3}^{1}+\delta \varepsilon x_{3}^{2}+\delta(1-2 \varepsilon) x_{3}^{3}=z_{3}$. Since Player 3 accepts proposals in state 1 if and only if $v_{3} \geq z_{3}$, this shows that the one-shot deviation property is satisfied.

Consider a history in state 1 after which Player 2 has to respond to a proposal $v$. Suppose first that $v_{3} \geq z_{3}$. A calculation similar to that in the previous paragraph shows that rejection of $v$ by Player 2 yields Player 2 a payoff of $z_{2}$. Acceptance yields $v_{2}$ because $v$ is accepted by Player 3. Hence accepting $v$ if and only if $v_{2} \geq z_{2}$ does not violate the one-shot deviation principle.

Suppose now that $v_{3}<z_{3}$. As before, rejecting $v$ by Player 2 gives payoff $z_{2}$. If Player 2 accepts $v$, then it is rejected by Player 3 and yields $\delta \varepsilon x_{2}^{1}+\delta \varepsilon x_{2}^{2}+\delta(1-2 \varepsilon) x_{2}^{3}=y_{2}$. It holds that $y_{2}<z_{2}$ since

$z_{2}-y_{2}=\frac{\delta(1-\delta)(1-3 \varepsilon)}{1-\delta+2 \delta \varepsilon+\delta^{2} \varepsilon-3 \delta^{2} \varepsilon^{2}}>0$.

Thus rejecting $v$ does not violate the one-shot deviation principle.

The verification of the one-shot deviation property for Player 1 is trivial for histories where he responds. We have already argued that $z_{2}+z_{3}>1$ if $\delta>1 /(2-6 \varepsilon)$. For such values of $\delta$, Player 1 cannot make a profitable one-shot deviation as a proposer.

Case 2. Decision nodes in state 2.

Using the same argument as in the proof of the previous proposition, we can show that the one-shot deviation property is satisfied.

Consider a history in state 2 after which Player 2 proposes. Since the proposal $\tilde{\theta}^{2}$ of Player 2 gives Players 1 and 3 the least amount they are willing to accept, there is no profitable one-shot deviation for Player 2 which will be accepted by Players 1 and 3. Consider a one-shot deviation by Player 2 which is rejected by some player. Ultimately, such a proposal leads to breakdown and payoff 0 for Player 2, or an acceptance of $\tilde{\theta}^{2}$ and payoff $1-y_{3}$ for Player 2 or an acceptance of $\tilde{\theta}^{3}$ and Payoff $y_{2}$ for Player 2 . Since it is easily verified that $y_{2}<1-y_{3}$, the expected payoff for Player 2 is less than $x_{2}^{2}=1-y_{3}$, so the deviation is not profitable.

Case 3. Decision nodes in state 3 .

This case is analogous to the Case 2 .

Notice that the strategy profile $(\tilde{\theta}, \tilde{A})$ violates no delay and efficient proposals which are two of the properties listed in Theorem 3.4. This is because there is no agreement in state 1 , and because the proposal $\tilde{\theta}^{1}$, which is indeterminate, may be inefficient. Proposition 5.3 yields limit equilibria of the form $\left(\theta^{1}, \theta^{2}, \theta^{3}\right)$ where $\theta^{1} \in V$ with $\theta_{1}^{1} \geq 0$ and

$\theta^{2}=\theta^{3}=\left(0, \frac{2-3 \varepsilon}{3-3 \varepsilon}, \frac{1}{3-3 \varepsilon}\right)$.

Clearly, not all players make the same proposals in the limit. The fact that in both cases Players 2 and 3 do make the same proposals in the limit can be deduced from Theorem 3.4. Indeed, since Player 1's proposal is rejected, one can view the resulting system, involving Players 2 and 3 only, as an exogenous protocol.

\section{Non-existence of SSPEs}

In the examples of the previous two sections, all properties of Theorem 3.4 are violated, with the exception of the existence of an SSPE. In this section, we will present an example where no SSPE exists at all, neither one with immediate agreement, nor one with delay. The only modification when compared to Example 4.1 is that in state 1 , after a rejection by Player 2 or 3 , we follow the rejectorproposes protocol with probability $1 / 2$ and return to state 1 with the complementary probability.

Example 6.1. There are three players and three states, $S=N=$ $\{1,2,3\}$. Each player is the proposer in one state and players respond in ascending order, so we have

$\iota(1)=\left(1, \pi^{0}\right)$,

$\iota(2)=\left(2, \pi^{0}\right)$,

$\iota(3)=\left(3, \pi^{0}\right)$,

where $\pi^{0}$ is the identity. Players have to divide a surplus of one unit, $V=\left\{v \in \mathbb{R}^{3} \mid v_{1}+v_{2}+v_{3} \leq 1\right\}$. In state $s=1$, the transitions depend on the identity of the player who rejects a proposal,

$p^{1}(1)=\left(\frac{1}{3}, \frac{1}{3}, \frac{1}{3}\right)$,

$p^{2}(1)=\left(\frac{1}{2}, \frac{1}{2}, 0\right)$,

$p^{3}(1)=\left(\frac{1}{2}, 0, \frac{1}{2}\right)$.

States $s=2,3$ are absorbing.

$p^{i}(2)=(0,1,0), \quad i \in N$,

$p^{i}(3)=(0,0,1), \quad i \in N$

Proposition 6.2. For $\delta \in(2 / 3,3 / 4)$, there is no SSPE in Example 6.1.

Proof. Suppose $(\theta, A)$ is an SSPE. It clearly holds that $\theta^{2}=$ $(0,1,0)$ and $\theta^{3}=(0,0,1)$. Now let $x=u(\theta, A \mid 1)$ be the equilibrium utilities conditional on state 1 and let $z^{i}$ be the vector of continuation payoffs after a proposal in state 1 is rejected by Player $i$. We have

$z^{1}=\frac{\delta}{3} x+\frac{\delta}{3}(0,1,0)+\frac{\delta}{3}(0,0,1)$,

$z^{2}=\frac{\delta}{2} x+\frac{\delta}{2}(0,1,0)$,

$z^{3}=\frac{\delta}{2} x+\frac{\delta}{2}(0,0,1)$

We distinguish four possible cases.

Case 1. $\theta^{1}$ is accepted by Players 1,2 , and 3 .

In this case it holds that $x=\theta^{1}$. Since all players accept $\theta^{1}$, we have

$x_{1} \geq z_{1}^{1}=\frac{\delta}{3} x_{1}$,

$x_{2} \geq z_{2}^{2}=\frac{\delta}{2} x_{2}+\frac{\delta}{2}$,

$x_{3} \geq z_{3}^{3}=\frac{\delta}{2} x_{3}+\frac{\delta}{2}$.

This gives the inequalities $x_{1} \geq 0, x_{2} \geq \delta /(2-\delta)$, and $x_{3} \geq$ $\delta /(2-\delta)$, whereas at the same time $x_{1}+x_{2}+x_{3}=\theta_{1}^{1}+\theta_{2}^{1}+\theta_{3}^{1} \leq 1$. For $\delta>2 / 3$, we have a contradiction.

Case 2. $\theta^{1}$ is accepted by Players 1 and 2, and rejected by Player 3 . 
Since Player 3 rejects $\theta^{1}$, we have $x=z^{3}$, and in particular, $x_{2}=$ $z_{2}^{3}=(\delta / 2) x_{2}$. Since $\delta / 2<1$, it follows that $z_{2}^{3}=x_{2}=0$. But Player 2 accepts, so it must hold that $z_{2}^{3}=0 \geq z_{2}^{2}=\delta / 2$, contradicting that $\delta$ is strictly positive.

Case 3. $\theta^{1}$ is accepted by Player 1 and rejected by Player 2 .

Since Player 2 rejects $\theta^{1}$, we have $x=z^{2}$, from which it follows that $x=(0, \delta /(2-\delta), 0)$ and in particular that $z_{2}^{2}=\delta /(2-\delta)$. Plugging $x_{1}=0$ into the equation for $z^{1}$ and $x_{3}=0$ into the equation for $z^{3}$ gives $z_{1}^{1}=0$ and $z_{3}^{3}=\delta / 2$, respectively. Since $z_{1}^{1}+z_{2}^{2}+z_{3}^{3}=0+\delta / 2+\delta /(2-\delta)<1$ if $\delta<3 / 4$, there exists a proposal $v \in V$ such that $v \gg\left(z_{1}^{1}, z_{2}^{2}, z_{3}^{3}\right)$. Clearly, $v$ would be accepted by all players. Since $v_{1}>x_{1}=0$, proposing $v$ instead of $\theta^{1}$ would be a profitable deviation for Player 1 .

Case 4. $\theta^{1}$ is rejected by Player 1 .

If Player 1 rejects his own proposal, then $x=z^{1}$, so $x=$ $(0, \delta /(3-\delta), \delta /(3-\delta))$, and in particular $z_{1}^{1}=0$. Plugging the expression for $x$ into the equations for $z^{2}$ and $z^{3}$, we find that $z_{2}^{2}=z_{3}^{3}=3 \delta /(6-2 \delta)$. Given that $\delta<3 / 4$, we have that $z_{1}^{1}+z_{2}^{2}+z_{3}^{3}<1$, thus there exists a proposal $v \in V$ such that $v \gg\left(z_{1}^{1}, z_{2}^{2}, z_{3}^{3}\right)$. The proposal $v$ would be accepted unanimously. Since $v_{1}>x_{1}=0$, it would be a profitable deviation for Player 1 to propose $v$ instead of $\theta^{1}$.

\section{Robustness of Example 6.1}

In this section, we will examine the robustness of Example 6.1 to perturbations of the transition probabilities. In particular, we demonstrate that the presence of absorbing states is not crucial for the lack of SSPEs. Consider Example 6.1 and perturb all transition probabilities by $\varepsilon \in[0,1 / 3)$. We obtain the following example.

Example 7.1. There are three players and three states, $S=N=$ $\{1,2,3\}$. Each player is the proposer in one state and players respond in ascending order, so we have
$\iota(1)=\left(1, \pi^{0}\right)$,
$\iota(2)=\left(2, \pi^{0}\right)$,
$\iota(3)=\left(3, \pi^{0}\right)$,

where $\pi^{0}$ is the identity. Players have to divide a surplus of one unit, $V=\left\{v \in \mathbb{R}^{3} \mid v_{1}+v_{2}+v_{3} \leq 1\right\}$. In state $s=1$, the transitions depend on the identity of the player who rejects a proposal,

$p^{1}(1)=\left(\frac{1}{3}, \frac{1}{3}, \frac{1}{3}\right)$

$p^{2}(1)=\left(\frac{1}{2}-\frac{\varepsilon}{2}, \frac{1}{2}-\frac{\varepsilon}{2}, \varepsilon\right)$,

$p^{3}(1)=\left(\frac{1}{2}-\frac{\varepsilon}{2}, \varepsilon, \frac{1}{2}-\frac{\varepsilon}{2}\right)$.

States $s=2,3$ are "almost absorbing", there is a small strictly positive probability to leave these states.

$p^{i}(2)=(\varepsilon, 1-2 \varepsilon, \varepsilon), \quad i \in N$,

$p^{i}(3)=(\varepsilon, \varepsilon, 1-2 \varepsilon), \quad i \in N$.

Clearly, Example 7.1 satisfies the strong regularity conditions of Assumption D.

Proposition 7.2. For every $\delta \in(2 / 3,3 / 4)$, there exists $\bar{\varepsilon}>0$ such that for every $\varepsilon \in[0, \bar{\varepsilon}]$ there is no SSPE in Example 7.1.

Proof. Suppose $(\theta, A)$ is an SSPE. Let $x=u(\theta, A \mid 1)$ be the equilibrium utilities conditional on state 1 and let $z^{i}$ be the vector of continuation payoffs after a proposal in state 1 is rejected by Player $i$.

We argue first that the proposals $\theta^{2}$ and $\theta^{3}$ are going to be accepted. Suppose, to the contrary, that $\theta^{2}$ is rejected. Since transition probabilities in state 2 do not depend on the identity of the rejector, we find that the vector of continuation payoffs conditional on the rejection by a player in state 2 , say $y$, is independent of the identity of the rejecting player. Moreover, since negotiations break down with probability $1-\delta$, it holds that $\sum_{i \in N} y_{i} \leq \delta$. Player 2 can therefore make a proposal giving every player a payoff strictly above his continuation payoff following a rejection. Such a proposal would be accepted, so Player 2 has a profitable deviation, which leads to a contradiction. Consequently, $\theta^{2}$ is going to be accepted. Using exactly the same argument, it follows that $\theta^{3}$ is going to be accepted.

We have

$z^{1}=\frac{\delta}{3} x+\frac{\delta}{3} \theta^{2}+\frac{\delta}{3} \theta^{3}$

$z^{2}=\frac{\delta-\delta \varepsilon}{2} x+\frac{\delta-\delta \varepsilon}{2} \theta^{2}+\delta \varepsilon \theta^{3}$,

$z^{3}=\frac{\delta-\delta \varepsilon}{2} x+\delta \varepsilon \theta^{2}+\frac{\delta-\delta \varepsilon}{2} \theta^{3}$.

The continuation payoff of Player 3 conditional on his rejection in state 2 is equal to $\delta(1-2 \varepsilon) \theta_{3}^{2}+\delta \varepsilon\left(x_{3}+\theta_{3}^{3}\right)$. Player 3 therefore accepts any proposal giving a strictly higher payoff. Since Player 2 should not have a profitable deviation, we find that

$\theta_{3}^{2}=\delta(1-2 \varepsilon) \theta_{3}^{2}+\delta \varepsilon\left(x_{3}+\theta_{3}^{3}\right)$.

By rearranging terms, we find that

$\theta_{3}^{2}=\frac{\delta \varepsilon\left(x_{3}+\theta_{3}^{3}\right)}{1-\delta+2 \delta \varepsilon} \leq \frac{2 \delta \varepsilon}{1-\delta+2 \delta \varepsilon}$

By a similar argument, we find that

$\theta_{1}^{2}=\frac{\delta \varepsilon\left(x_{1}+\theta_{1}^{3}\right)}{1-\delta+2 \delta \varepsilon} \leq \frac{2 \delta \varepsilon}{1-\delta+2 \delta \varepsilon}$.

We can apply the same reasoning in state 3 , to obtain

$\theta_{1}^{3} \leq \frac{2 \delta \varepsilon}{1-\delta+2 \delta \varepsilon}$

$\theta_{2}^{3} \leq \frac{2 \delta \varepsilon}{1-\delta+2 \delta \varepsilon}$

and it follows that

$\theta_{2}^{2} \geq \frac{1-\delta-2 \delta \varepsilon}{1-\delta+2 \delta \varepsilon}$

$\theta_{3}^{3} \geq \frac{1-\delta-2 \delta \varepsilon}{1-\delta+2 \delta \varepsilon}$.

Let some $\delta \in(2 / 3,3 / 4)$ be given. We distinguish four cases.

Case $1 . \theta^{1}$ is accepted by Players 1,2 , and 3 .

In this case $x=\theta^{1}$. Since all players accept $\theta^{1}$, we have

$$
\begin{aligned}
x_{1} \geq & z_{1}^{1} \geq \\
x_{2} \geq & z_{2}^{2} \geq \frac{\delta-\delta \varepsilon}{2} x_{2}+\frac{\delta-\delta \varepsilon}{2} \theta_{2}^{2} \geq \frac{\delta-\delta \varepsilon}{2} x_{2} \\
& +\frac{\delta-\delta \varepsilon}{2} \frac{1-\delta-2 \delta \varepsilon}{1-\delta+2 \delta \varepsilon}, \\
x_{3} \geq & z_{3}^{3} \geq \frac{\delta-\delta \varepsilon}{2} x_{3}+\frac{\delta-\delta \varepsilon}{2} \theta_{3}^{3} \geq \frac{\delta-\delta \varepsilon}{2} x_{3} \\
& +\frac{\delta-\delta \varepsilon}{2} \frac{1-\delta-2 \delta \varepsilon}{1-\delta+2 \delta \varepsilon} .
\end{aligned}
$$


Rearranging terms, we find that

$x_{1} \geq 0$,

$x_{2} \geq \frac{\delta-\delta \varepsilon}{2-\delta+\delta \varepsilon} \frac{1-\delta-2 \delta \varepsilon}{1-\delta+2 \delta \varepsilon}$,

$x_{3} \geq \frac{\delta-\delta \varepsilon}{2-\delta+\delta \varepsilon} \frac{1-\delta-2 \delta \varepsilon}{1-\delta+2 \delta \varepsilon}$,

SO

$x_{1}+x_{2}+x_{3} \geq \frac{2 \delta-2 \delta \varepsilon}{2-\delta+\delta \varepsilon} \frac{1-\delta-2 \delta \varepsilon}{1-\delta+2 \delta \varepsilon}$.

If we evaluate the right-hand side of (1) at $\varepsilon=0$, we find it to be equal to $2 \delta /(2-\delta)$, which is strictly above 1 since $\delta \in(2 / 3,3 / 4)$. Since the right-hand side of (1) is continuous in $\varepsilon$, there is $\bar{\varepsilon}_{1}>0$ such that for every $\varepsilon \in\left[0, \bar{\varepsilon}_{1}\right)$ it holds that the expression in (1) is strictly above 1 . At the same time it holds that $x_{1}+x_{2}+x_{3}=$ $\theta_{1}^{1}+\theta_{2}^{1}+\theta_{3}^{1} \leq 1$, so we have a contradiction.

Case 2. $\theta^{1}$ is accepted by Players 1 and 2 , and rejected by Player 3 .

Since Player 3 rejects $\theta^{1}$, we have

$x_{2}=z_{2}^{3}=\frac{\delta-\delta \varepsilon}{2} x_{2}+\delta \varepsilon \theta_{2}^{2}+\frac{\delta-\delta \varepsilon}{2} \theta_{2}^{3}$.

By rearranging terms, we find that

$$
\begin{aligned}
z_{2}^{3} & =\frac{2 \delta \varepsilon}{2-\delta+\delta \varepsilon} \theta_{2}^{2}+\frac{\delta-\delta \varepsilon}{2-\delta+\delta \varepsilon} \theta_{2}^{3} \\
& \leq \frac{2 \delta \varepsilon}{2-\delta+\delta \varepsilon}+\frac{\delta-\delta \varepsilon}{2-\delta+\delta \varepsilon} \frac{2 \delta \varepsilon}{1-\delta+2 \delta \varepsilon} .
\end{aligned}
$$

Since Player 2 accepts $\theta^{1}$, it holds that

$$
\begin{aligned}
z_{2}^{3} & \geq z_{2}^{2}=\frac{\delta-\delta \varepsilon}{2} x_{2}+\frac{\delta-\delta \varepsilon}{2} \theta_{2}^{2}+\delta \varepsilon \theta_{2}^{3} \\
& \geq \frac{\delta-\delta \varepsilon}{2} \frac{1-\delta-2 \delta \varepsilon}{1-\delta+2 \delta \varepsilon} .
\end{aligned}
$$

Evaluating (2) and (3) at $\varepsilon=0$, we find that $z_{2}^{3}=0$ and $z_{2}^{2}=$ $\delta / 2>1 / 3$, a contradiction to $z_{2}^{3} \geq z_{2}^{2}$. Since the expressions in (2) and (3) are continuous in $\varepsilon$, there is $\bar{\varepsilon}_{2}>0$ such that we obtain the same contradiction for every $\varepsilon \in\left[0, \bar{\varepsilon}_{2}\right]$.

Case 3. $\theta^{1}$ is accepted by Player 1 and rejected by Player 2 .

Since Player 2 rejects $\theta^{1}$, we have

$x=z^{2}=\frac{\delta-\delta \varepsilon}{2} x+\frac{\delta-\delta \varepsilon}{2} \theta^{2}+\delta \varepsilon \theta^{3}$,

so

$$
\begin{aligned}
x_{1} & =z_{1}^{2}=\frac{\delta-\delta \varepsilon}{2-\delta+\delta \varepsilon} \theta_{1}^{2}+\frac{2 \delta \varepsilon}{2-\delta+\delta \varepsilon} \theta_{1}^{3} \\
& \leq \frac{\delta+\delta \varepsilon}{2-\delta+\delta \varepsilon} \frac{2 \delta \varepsilon}{1-\delta+2 \delta \varepsilon}, \\
x_{2} & =z_{2}^{2}=\frac{\delta-\delta \varepsilon}{2-\delta+\delta \varepsilon} \theta_{2}^{2}+\frac{2 \delta \varepsilon}{2-\delta+\delta \varepsilon} \theta_{2}^{3} \\
& \leq \frac{\delta-\delta \varepsilon}{2-\delta+\delta \varepsilon}+\frac{2 \delta \varepsilon}{2-\delta+\delta \varepsilon} \frac{2 \delta \varepsilon}{1-\delta+2 \delta \varepsilon},
\end{aligned}
$$$$
x_{3}=z_{3}^{2}=\frac{\delta-\delta \varepsilon}{2-\delta+\delta \varepsilon} \theta_{3}^{2}+\frac{2 \delta \varepsilon}{2-\delta+\delta \varepsilon} \theta_{3}^{3}
$$$$
\leq \frac{\delta-\delta \varepsilon}{2-\delta+\delta \varepsilon} \frac{2 \delta \varepsilon}{1-\delta+2 \delta \varepsilon}+\frac{2 \delta \varepsilon}{2-\delta+\delta \varepsilon} \text {. }
$$

We use these expressions to obtain

$$
\begin{aligned}
z_{1}^{1}= & \frac{\delta}{3} x_{1}+\frac{\delta}{3} \theta_{1}^{2}+\frac{\delta}{3} \theta_{1}^{3} \leq \frac{\delta}{3} \frac{\delta+\delta \varepsilon}{2-\delta+\delta \varepsilon} \frac{2 \delta \varepsilon}{1-\delta+2 \delta \varepsilon} \\
& +\frac{\delta}{3} \frac{4 \delta \varepsilon}{1-\delta+2 \delta \varepsilon},
\end{aligned}
$$

$$
\begin{aligned}
z_{3}^{3}= & \frac{\delta-\delta \varepsilon}{2} x_{3}+\delta \varepsilon \theta_{3}^{2}+\frac{\delta-\delta \varepsilon}{2} \theta_{3}^{3} \leq \frac{\delta-\delta \varepsilon}{2} \frac{\delta-\delta \varepsilon}{2-\delta+\delta \varepsilon} \\
& \times \frac{2 \delta \varepsilon}{1-\delta+2 \delta \varepsilon}+\frac{\delta-\delta \varepsilon}{2} \frac{2 \delta \varepsilon}{2-\delta+\delta \varepsilon} \\
& +\delta \varepsilon \frac{2 \delta \varepsilon}{1-\delta+2 \delta \varepsilon}+\frac{\delta-\delta \varepsilon}{2} .
\end{aligned}
$$

Evaluating the upper bounds for $x_{1}, z_{1}^{1}, z_{2}^{2}$, and $z_{3}^{3}$ at $\varepsilon=0$ gives values equal to $0,0, \delta /(2-\delta)$, and $\delta / 2$, respectively, so an upper bound for $z_{1}^{1}+z_{2}^{2}+z_{3}^{3}$ is given by $\delta /(2-\delta)+\delta / 2<39 / 40$ since $\delta<$ $3 / 4$. Since the expressions for the upper bounds on $x_{1}, z_{1}^{1}, z_{2}^{2}$, and $z_{3}^{3}$ are continuous in $\varepsilon$, there is $\bar{\varepsilon}_{3}>0$ such that for every $\varepsilon \in\left[0, \bar{\varepsilon}_{3}\right]$ it holds that $x_{1}<1 / 40$ and $z_{1}^{1}+z_{2}^{2}+z_{3}^{3}<39 / 40$. For these values of $\varepsilon$ there exists a proposal $v \in V$ such that $v_{1} \geq 1 / 40$ and $v \gg$ $\left(z_{1}^{1}, z_{2}^{2}, z_{3}^{3}\right)$. Such a proposal $v$ would be accepted unanimously and satisfies $v_{1}>x_{1}$, so would be a profitable deviation for Player 1 .

Case 4. $\theta^{1}$ is rejected by Player 1 .

If Player 1 rejects his own proposal, then

$x=z^{1}=\frac{\delta}{3} x+\frac{\delta}{3} \theta^{2}+\frac{\delta}{3} \theta^{3}$,

so

$x_{1}=z_{1}^{1}=\frac{\delta}{3-\delta} \theta_{1}^{2}+\frac{\delta}{3-\delta} \theta_{1}^{3} \leq \frac{\delta}{3-\delta} \frac{4 \delta \varepsilon}{1-\delta+2 \delta \varepsilon}$,

$x_{2}=z_{2}^{1}=\frac{\delta}{3-\delta} \theta_{2}^{2}+\frac{\delta}{3-\delta} \theta_{2}^{3} \leq \frac{\delta}{3-\delta}+\frac{\delta}{3-\delta} \frac{2 \delta \varepsilon}{1-\delta+2 \delta \varepsilon}$,

$x_{3}=z_{3}^{1}=\frac{\delta}{3-\delta} \theta_{3}^{2}+\frac{\delta}{3-\delta} \theta_{3}^{3} \leq \frac{\delta}{3-\delta} \frac{2 \delta \varepsilon}{1-\delta+2 \delta \varepsilon}+\frac{\delta}{3-\delta}$.

It now follows that

$$
\begin{aligned}
z_{2}^{2}= & \frac{\delta-\delta \varepsilon}{2} x_{2}+\frac{\delta-\delta \varepsilon}{2} \theta_{2}^{2}+\delta \varepsilon \theta_{2}^{3} \leq \frac{\delta-\delta \varepsilon}{2} \frac{\delta}{3-\delta} \\
& +\frac{\delta-\delta \varepsilon}{2} \frac{\delta}{3-\delta} \frac{2 \delta \varepsilon}{1-\delta+2 \delta \varepsilon}+\frac{\delta-\delta \varepsilon}{2}+\delta \varepsilon \frac{2 \delta \varepsilon}{1-\delta+2 \delta \varepsilon}, \\
z_{3}^{3}= & \frac{\delta-\delta \varepsilon}{2} x_{3}+\delta \varepsilon \theta_{3}^{2}+\frac{\delta-\delta \varepsilon}{2} \theta_{3}^{3} \\
\leq & \frac{\delta-\delta \varepsilon}{2} \frac{\delta}{3-\delta} \frac{2 \delta \varepsilon}{1-\delta+2 \delta \varepsilon}+\frac{\delta-\delta \varepsilon}{2} \frac{\delta}{3-\delta} \\
& +\delta \varepsilon \frac{2 \delta \varepsilon}{1-\delta+2 \delta \varepsilon}+\frac{\delta-\delta \varepsilon}{2} .
\end{aligned}
$$

Evaluating the upper bounds for $z_{1}^{1}, z_{2}^{2}$, and $z_{3}^{3}$ at $\varepsilon=0$ gives values equal to $0,3 \delta /(6-2 \delta)$, and $3 \delta /(6-2 \delta)$, respectively, so an upper bound for $z_{1}^{1}+z_{2}^{2}+z_{3}^{3}$ is given by $6 \delta /(6-2 \delta)<1$ since $\delta<3 / 4$. Since the expressions for the upper bounds on $z_{1}^{1}, z_{2}^{2}$, and $z_{3}^{3}$ are continuous in $\varepsilon$, there is $\bar{\varepsilon}_{4}>0$ such that for every $\varepsilon \in\left[0, \bar{\varepsilon}_{4}\right]$ it holds that $z_{1}^{1}+z_{2}^{2}+z_{3}^{3}<1$. For these values of $\varepsilon$ there exists a proposal $v \in V$ such that $v \gg\left(z_{1}^{1}, z_{2}^{2}, z_{3}^{3}\right)$. Such a proposal $v$ would be accepted unanimously and satisfies $v_{1}>z_{1}^{1}=x_{1}$, so would be a profitable deviation for Player 1 .

For $\bar{\varepsilon}=\min \left\{\bar{\varepsilon}_{1}, \bar{\varepsilon}_{2}, \bar{\varepsilon}_{3}, \bar{\varepsilon}_{4}\right\}>0$, for every $\varepsilon \leq \bar{\varepsilon}$, there is no SSPE in Example 7.1.

\section{Coalitional bargaining}

An interesting direction for future research concerns the extension of our analysis to the framework of coalitional bargaining. The simplest set-up would be the one of transferable utility games, where any coalition can generate some surplus. Chatterjee et al. (1993) consider the rejector-proposes protocol for this framework and give examples to show that SSPE may exhibit delay and may fail to have efficient proposals. They show that strict convexity of the transferable utility game is sufficient to get convergence to the egalitarian allocation of Dutta and Ray (1989) if the common discount factor converges to one. Kawamori (2008) studies 
an endogenous protocol with a general probabilistic selection of a new proposer, conditional on who rejects the current proposal. It is shown that the possibility of the existence of an SSPE with efficient proposals decreases as the probability that the rejector in the preceding round becomes the proposer increases.

Okada (1996) considers the protocol with time-invariant uniform recognition probabilities and shows that an SSPE does not exhibit delay if the transferable utility game is superadditive. To obtain an SSPE with efficient proposals, the grand coalition should have the largest per capita value among all coalitions. Moreover, under this condition, as the common discount factor goes to one, the value of the grand coalition is divided equally among the players.

Compte and Jehiel (2010) and Okada (2011) show how the use of mixed strategies leads to a general SSPE existence result. For the protocol with time-invariant uniform recognition probabilities, Compte and Jehiel (2010) provide both necessary and sufficient conditions, which are stronger than non-emptiness of the core, to get convergence to the coalitional Nash bargaining solution if at most one coalition can actually form.

\section{Conclusion}

We consider bargaining games of perfect information with a unanimous acceptance rule. The focus of the analysis is on the influence of the bargaining protocol on the bargaining outcomes. We consider a framework where the proposer and the order of the responding players is determined by a state variable. The probability distribution over states in the following period is determined jointly by the current state and the identity of the player who rejected the previous proposal.

Many papers in the existing literature are incorporated as special cases of this framework. It is either assumed that the identity of the future proposer only depends on the identity of the current proposer or it is assumed that it only depends on the identity of the rejector. In both cases it holds that SSPEs exist, have the immediate acceptance property, and involve efficient proposals only. Asymptotically, as players become perfectly patient, all such equilibria converge to an appropriately defined asymmetric Nash bargaining solution.

In this paper, however, we show that these conclusions do not carry over to the general framework: SSPEs need not exist. When they do exist, they may exhibit delay and involve inefficient proposals. The limit equilibrium need not be unique, limit equilibrium utilities need not be unique, and players may make different equilibrium proposals in the limit.

The central message in Britz et al. (2014) is that the bargaining power of a player is determined by the probability to propose conditional on his own rejection. For the more general framework considered here, we show that even though players can propose with positive probability conditional on their own rejection, they might still have no bargaining power at all.

\section{References}

Ausubel, L.M., Cramton, P., Deneckere, R.J., 2002. Bargaining with incomplete information. In: Aumann, R.J., Hart, S. (Eds.), Handbook of Game Theory, Vol. 3. Elsevier Science Publishers, Amsterdam, pp. 1897-1945.

Banks, J., Duggan, J., 2000. A bargaining model of collective choice. Am. Polit. Sci. Rev. 94, 73-88.

Binmore, K., 1987. Perfect equilibria in bargaining models. In: Binmore, K., Dasgupta, P. (Eds.), The Economics of Bargaining. Basil Blackwell, Oxford, UK, pp. 77-105.

Binmore, K., Rubinstein, A., Wolinsky, A., 1986. The Nash bargaining solution in economic modelling. Rand J. Econ. 17, 176-188.

Bloch, F., 1996. Sequential formation of coalitions in games with externalities and fixed payoff division. Games Econom. Behav. 14, 90-123.

Britz, V., Herings, P.J.J., Predtetchinski, A., 2010. Non-cooperative support for the asymmetric Nash bargaining solution. J. Econom. Theory 145, 1951-1967.

Britz, V., Herings, P.J.J., Predtetchinski, A., 2014. On the convergence to the Nash bargaining solution for action-dependent bargaining protocols. Games Econom. Behav. 86, 178-183.

Chatterjee, K., Dutta, B., Ray, D., Sengupta, K., 1993. A noncooperative theory of coalitional bargaining. Rev. Econom. Stud. 60, 463-477.

Compte, O., Jehiel, P., 2010. The coalitional Nash bargaining solution. Econometrica 78, 1593-1623.

Dutta, B., Ray, D., 1989. A concept of egalitarianism under participation constraints. Econometrica 57, 615-636.

Fudenberg, D., Tirole, J., 1991. Game Theory. MIT Press, Cambridge, Massachusetts.

Haller, H., 1986. Non-cooperative bargaining of $N \geq 3$ players. Econom. Lett. 22, $11-13$.

Hart, S., Mas-Colell, A., 1996. Bargaining and value. Econometrica 64, 357-380.

Herings, P.J.J., Predtetchinski, A., 2015. Bargaining with non-convexities. Games Econom. Behav. 90, 151-161.

Herrero, M.J., 1985. A strategic bargaining approach to market institutions (Ph.D. thesis), London School of Economics, pp. 1-111.

Jéhiel, P., Moldovanu, B., 1995. Cyclical delay in bargaining with externalities. Rev. Econom. Stud. 62, 619-637.

Kalandrakis, T., 2004. Equilibria in sequential bargaining games as solutions to systems of equations. Econom. Lett. 84, 407-411.

Kawamori, T., 2008. A note on selection of proposers in coalitional bargaining. Internat. J. Game Theory 37, 525-532.

Kultti, K., Vartiainen, H., 2010. Multilateral non-cooperative bargaining in a general utility space. Internat. J. Game Theory 39, 677-689.

Laruelle, A., Valenciano, F., 2008. Noncooperative foundations of bargaining power in committees and the Shapley-Shubik index. Games Econom. Behav. 63, 341-353.

Maskin, E.S., Tirole, J., 2001. Markov perfect equilibrium, I. Observable actions. J. Econom. Theory 100, 191-219.

Merlo, A., Wilson, C., 1995. A stochastic model of sequential bargaining with complete information. Econometrica 63, 371-399.

Miyakawa, T., 2008. Noncooperative foundation of $n$-person asymmetric Nash bargaining solution. J. Econ. Kwansei Gakuin Univ. 62, 1-18.

Okada, A., 1996. A noncooperative coalitional bargaining game with random proposers. Games Econom. Behav. 16, 97-108.

Okada, A., 2011. Coalitional bargaining games with random proposers: Theory and application. Games Econom. Behav. 73, 227-235.

Rubinstein, A., 1982. Perfect equilibrium in a bargaining model. Econometrica 50, 97-109.

Selten, R., 1981. A noncooperative model of characteristic function bargaining. In: Böhm, V., Nachtkamp, H.H. (Eds.), Essays in Game Theory and Mathematical Economics in Honor of Oskar Morgenstern. Bibliografisches Institut Mannheim, pp. 131-151.

Yildiz, M., 2003. Bargaining without a common prior: An immediate agreement theorem. Econometrica 71, 793-811. 